\title{
Distribución del Pequeño Escarabajo de la Colmena, Aethina tumida, en abejas africanizadas (Apis mellifera) en diferentes zonas apícolas de Costa Rica
}

\section{Distribution of small hive beetles, Aethina tumida, in Africanized honey bees (Apis mellifera) in different beekeeping areas of Costa Rica}

\section{Distribuição do pequeno besouro da colmeia, Aethina tumida, em abelhas africanizadas (Apis mellifera) em diferentes áreas apícolas da Costa Rica}

Martha Arguedas Mora; Juan Francisco Soto González; Marianyela Ramírez Montero; Rafael A. Calderón Fallas ${ }^{1,2 \otimes}$

1 Maestría en Apicultura Tropical, Centro de Investigaciones Apícolas Tropicales, Universidad Nacional, Heredia, Costa Rica. Correos electrónicos: marvet1094@gmail.com; manyeramirezm@gmail.com; juansg1986@hotmail.com

2 Programa Integrado de Patología Apícola, Centro de Investigaciones Apícolas Tropicales, Universidad Nacional, Heredia, Costa Rica. rafael.calderon.fallas@una.cr

Recibido: 8 de setiembre de 2020 Corregido: 15 de octubre de 2020 Aceptado: 23 de octubre de 2020

\begin{abstract}
Resumen
El primer reporte sobre la presencia del Pequeño Escarabajo de la Colmena (PEC), Aethina tumida, en Costa Rica, se realizó el 25 de agosto 2015, en La Cruz, Guanacaste; lo cual aumenta el riesgo de dispersión a otras zonas apícolas del país. Por tanto, para este estudio, se realizó un muestreo de mayo a octubre del 2019. Se analizó 305 colmenas, distribuidas en 61 apiarios, ubicados en San Ramón, Alajuela. Adicionalmente, se examinó muestras de escarabajos adultos provenientes de Ulloa, Heredia y de Potrerillos de Liberia, Guanacaste. En cada apiario se analizó, aleatoriamente, cinco colmenas. Se revisó la tapa, los marcos, las cajas y el piso, con el fin de identificar la presencia de huevos, larvas y/o adultos del escarabajo. También, para reconocer algún signo relacionado, como galerías en los panales, apariencia húmeda de las abejas, entre otros. Las muestras de escarabajos adultos se analizaron en el laboratorio de Patología Apícola del CINAT-UNA. Las 305 colmenas evaluadas, en San Ramón de Alajuela, resultaron negativas para el Pequeño Escarabajo. Por otra parte, se confirmó la presencia de $A$. tumida en una colonia establecida en una casa de habitación en Ulloa, Heredia; también, en un apiario ubicado en Potrerillos de Liberia, el cual se encuentra aproximadamente a $25 \mathrm{~km}$ del foco inicial en La Cruz, Guanacaste. Con base en lo expuesto, se puede indicar la presencia de A. tumida en colmenas de abejas africanizadas en diferentes zonas de Costa Rica, con una distribución limitada. Al considerar que el escarabajo adulto puede volar y desplazarse, junto a enjambres silvestres; además, la trashumancia de colmenas por parte del apicultor, se debe mantener una revisión periódica de los apiarios, dado que las condiciones actuales de ausencia, en la mayoría de colmenas analizadas en este estudio, pueden cambiar.
\end{abstract}

Palabras clave: Aethina tumida, Pequeño Escarabajo de la Colmena, zonas apícolas, abejas africanizadas

区 Autor para correspondencia: rafael.calderon.fallas@una.cr 


\begin{abstract}
The first report of Small Hive Beetles (SHB), Aethina tumida, in Costa Rica was made on August 25, 2015, in La Cruz, Guanacaste, which increased the risk of dispersal to other beekeeping areas in the country. Therefore, in this study 305 colonies belonging to 61 apiaries, located in San Ramón, Alajuela, Costa Rica, were sampled from May to October 2019. In addition, adult beetle samples were analyzed from Ulloa, Heredia and Potrerillos, Liberia, Guanacaste. Five colonies were randomly selected in every apiary. Broods, boxes, combs, and bottom boards were checked for beetle eggs, larvae and/or adults, and for any related signs such as galleries in the combs, and wet appearance of bees, among others. Adult beetles were analyzed at the Bee Pathology Lab of the Tropical Beekeeping Research Center (CINAT-UNA). The 305 colonies evaluated in San Ramón tested negative for SHBs. On the other hand, the presence of A. tumida was confirmed in a wild colony in a house located in Ulloa, Heredia and in an apiary in Potrerillos, Liberia, which is approximately 25 $\mathrm{km}$ from the initial beetle focus in La Cruz, Guanacaste. Based on the above, the presence of A. tumida can be confirmed in Africanized honey bee colonies in different beekeeping areas of Costa Rica, with a limited distribution. Given that adult SHB can fly and move with wild swarms and the transhumance of colonies by beekeepers, apiaries must be periodically checked because the current absence in most of the colonies analyzed in the present study can change.
\end{abstract}

Keywords: Aethina tumida, Small Hive Beetle, beekeeping areas, Africanized honey bees

\begin{abstract}
Resumo
O primeiro relato da ocorrência do Pequeno Besouro da Colmeia (PEC), Aethina tumida, na Costa Rica, foi feito em 25 de agosto de 2015, em La Cruz, Guanacaste; o que aumenta o risco de dispersão para outras áreas apícolas do país. Portanto, para este estudo, foi realizada uma amostragem de maio a outubro de 2019. Foram analisadas 305 colmeias, distribuídas em 61 apiários, localizados em San Ramón, Alajuela. Adicionalmente, amostras de besouros adultos de Ulloa, Heredia e Potrerillos de Liberia, Guanacaste foram examinadas. Em cada apiário, cinco colmeias foram analisadas aleatoriamente. Ninhadas, caixas, favos e tábuas de fundo foram verificados para identificar a presença de ovos, larvas e / ou adultos do besouro. Além disso, reconhecer quaisquer sinais relacionados, como galerias nos favos, o aspecto úmido das abelhas, entre outros. As amostras de besouros adultos foram analisadas no Laboratório de Patologia Apícola do CINAT-UNA. As 305 colmeias avaliadas, em San Ramón de Alajuela, foram negativas para o Besouro. Por outro lado, a presença de $A$. tumida foi confirmada em uma colônia instalada em uma casa em Ulloa, Heredia; também, em um apiário localizado em Potrerillos, Liberia, que fica a aproximadamente $25 \mathrm{~km}$ do foco inicial em La Cruz, Guanacaste. Com base no exposto, pode ser indicada a presença de A. tumida em colmeias africanizadas em diferentes áreas da Costa Rica, com uma distribuição limitada. Ao considerar que o besouro adulto pode voar e se mover, junto com os enxames selvagens; além da transumância das colmeias pelo apicultor, deve ser mantida uma revisão periódica dos apiários, visto que as atuais condições de ausência do besouro, na maioria das colmeias analisadas neste estudo, podem sofrer alterações.
\end{abstract}

Palavras-chave: Aethina tumida, pequeno besouro da colmeia, áreas de apicultura, abelhas africanizadas

\title{
Introducción
}

Se ha reportado más de 35 enfermedades que afectan a las abejas melíferas. La mayoría representa un verdadero impacto sobre la salud de las colonias y la actividad apícola mundial (Martínez et al. 2016). Diferentes agentes patógenos causan enfermedad en las abejas: como las bacterias Paenibacills larvae (Loque Americano) y Melissococcus plutonius (Loque Europeo), los hongos Aspergillus flavus y Aspergillus fumigatus (Cría Momificada) y Ascosphaera apis (Cría de Tiza). Asimismo, los virus representan un problema sanitario para las colonias, entre ellos el virus de la Parálisis y el virus de la 
Rev. Ciencias Veterinarias, Vol. 38, N² 2, [13-29], E-ISSN: 2215-4507, julio-diciembre, 2020

DOI: https://doi.org/10.15359/rcv.38-2.2

URL: https://www.revistas.una.ac.cr/index.php/veterinaria/index

Ciencias

Veterinarias

Cría Sacciforme. Por otra parte, parásitos como el ácaro Varroa destructor (Varroosis) y plagas como el Pequeño Escarabajo de la Colmena, Aethina tumida, causan daños considerables en los apiarios (Calvo et al. 2013).

El Pequeño Escarabajo de la Colmena (PEC), A. tumida (Coleoptera: Nitidulidae), es una plaga de las abejas melíferas, nativo de África subsahariana (Neumann \& Ellis 2008). El cual se considera una especie invasora en América, Australia, Europa y Asia (Neumann et al. 2016; Neumann et al. 2018), que ocasiona serios problemas a la apicultura, principalmente en colmenas de abejas de origen europeo en Norteamérica y Australia (Cuthbertson et al. 2013; Hayes et al. 2015).

El daño, en las colmenas, lo provocan tanto los escarabajos adultos como las larvas. Ambos estadios se alimentan de miel y polen. No obstante, las larvas causan el mayor daño en la colonia, pues se alimentan de la cría de las abejas; forman galerías en los panales y ocasionan la fermentación de la miel, debido a la levadura Kodamea ohmeri, presente en sus deyecciones (Villalobos et al. 2014; Hayes et al. 2015). Se conoce que $A$. tumida puede reproducirse en hospederos alternos, como colonias de abejas nativas sin aguijón (meliponinos) y abejorros (Bombus sp.), así como en una gran variedad de frutas y en carne (FERA 2015; Neumann et al. 2018).

Debido al impacto negativo, que podría causar A. tumida en abejas africanizadas de Costa Rica, es de suma importancia monitorear apiarios de diferentes zonas apícolas, con el fin de determinar su presencia y distribución e informar al sector apícola para prevenir la pérdida de colmenas. Por consiguiente, el objetivo del estudio consiste en evaluar la presencia Aethina tumida en colmenas de abejas africanizadas en el cantón de San Ramón, Alajuela y en otras regiones del país.

Origen y distribución: El Pequeño Escarabajo, A. tumida, es un coleóptero de la familia Nitidulidae, descrito, por primera vez, en 1867, (Murray 1867) endémico de África del Sur o África subsahariana (Neumann 2004). Su distribución natural ocurre en la parte tropical y subtropical, en países como: Sudáfrica, Botsuana, Namibia, Zimbabue, Angola, Zambia, Tanzania y República Democrática del Congo (Hepburn \& Radloff 1998; Ellis 2003). Aunque algunos estudios indican que también podría encontrarse en zonas templadas (Sanford 2005; Torto et al. 2007). En la región de origen, este escarabajo no se considera una plaga de importancia económica, pues el éxito reproductivo se limita a colonias que se encuentran muy débiles, enfermas, estresadas o en nidos de abejas recientemente abandonados, siendo menos común en colonias fuertes (con una alta población de abejas) (Neumann 2004; Neumann \& Elzen 2004). La abeja africana (A. m. scutellata), tiene la capacidad de eliminar, de la colmena, los huevos y larvas del escarabajo. Lo indicado, permite controlar la población de A. tumida de forma más eficiente, comparado con abejas de origen europeo (Neumann \& Hartel 2004), en cuyas colmenas se ha observado infestaciones severas, con presencia de gran cantidad de larvas (Calderón et al. 2006).

Este escarabajo se reportó, por primera vez, en los Estados Unidos, en 1998, en colmenas de abejas europeas, ubicadas en Florida (Elzen et al. 1999). Posterior a su detección en Florida, se ha dispersado a diferentes estados como: Georgia, Carolina del Norte, Minnesota, Ohio, Pensilvania y New Jersey (Roldán 2014). Después, se extendió a Texas y California (Ellis et al. 2007). Se calcula que esta plaga causa pérdidas anuales de aproximadamente tres millones de dólares, en la industria apícola en los Estados Unidos (Hayes et al. 2015). 
Actualmente, A. tumida se encuentra distribuido en diversos países del mundo, como: Egipto (2000), Australia (2002), Canadá (2002), México (2007), Cuba (2012) y Brasil (2015) (Faucon 2003; Neumann \& Ellis 2008; OIE 2016; Idrissou et al. 2019). En Europa, la Administración Italiana declaró haberlo detectado en Calabria y Sicilia (2014) (Roldán 2014). En el caso de Portugal, se dio una alerta durante el verano del 2004, al encontrarse dos larvas en un envío de reinas, procedentes de un criadero de Texas. Se destruyó las colmenas, se desinfectó el suelo alrededor en ellas, se revisaron los envíos de ese criadero a otros países de Europa, como Francia y no se ha reportado más detecciones (Roldán 2014; Saldaña et al. 2014).

Situación en Centroamérica: A. tumida fue detectado, por primera vez, en El Salvador, en el 2013 (OIE, 2013). Posteriormente, se determinó su presencia en San Juan Sur, Rivas de Nicaragua, en marzo del 2014 (Calderón et al. 2014). Con la detección del escarabajo, en la zona fronteriza de Nicaragua, se generó una alerta en Costa Rica. Se inició un muestreo en 5 de las 7 provincias (Guanacaste, Puntarenas, Alajuela, San José y Heredia). Se abarcó las zonas con mayor concentración de colmenas en cada una de las provincias, para tener datos representativos de la situación del escarabajo en Costa Rica. Este muestreo se realizó en un extenso periodo que abarcó desde el 2014 hasta el 2017. Las muestras se analizaron en el laboratorio de Patología Apícola del CINAT-UNA, confirmando la presencia de $A$. tumida únicamente en un apiario centinela ubicado en Santa Cecilia, La Cruz, Guanacaste el 25 agosto del 2015 (Ramírez \& Calderón 2018).

Biología y ciclo de vida: Este escarabajo pertenece a la familia Nitidulidae. Gran parte de las especies, de esta familia, se alimentan de: frutas frescas o en descomposición, savia de las plantas, hongos, néctar y polen (Solís 2002; Neumann 2004; Stedman 2006). Diferentes estudios mencionan que, A. tumida se alimenta y reproduce en frutas como: melón, piña y banano. Sin embargo, su principal hospedero es la colonia de abejas melíferas. También, puede reproducirse en panales almacenados y en otros materiales apícolas (Neumann \& Elzen 2004; Cuthbertson et al. 2013; FERA 2015).

El primer signo de infestación, en las colonias, es la presencia de escarabajos adultos. En infestaciones severas, se observan larvas y miel fermentada (Calderón et al. 2006; FERA 2015). Por tanto, el apicultor debe revisar los rincones de la cámara de cría, uniones de los marcos con la caja y el fondo, en busca de algún signo de infestación (MAG 2012). El ciclo de vida, de este escarabajo, se divide en cuatro etapas: huevo, larva, pupa y adulto. Su desarrollo tarda aproximadamente entre cuatro a seis semanas, lo que da origen a seis generaciones por año. Se indica que, en zonas cálidas, los tiempos de desarrollo podrían reducirse (Stedman 2006).

Condiciones que benefician el desarrollo: El escarabajo A. tumida tiene una gran adaptabilidad a distintos ambientes. Se ha encontrado desde climas tropicales hasta fríos. Además, es capaz de retrasar su desarrollo de acuerdo con las condiciones del medio y el alimento disponible (Saldaña et al. 2014; Argueta 2015). Entre los factores que benefician su desarrollo, se puede indicar la humedad, la cual debe ser mayor al $50 \%$ para la eclosión de los huevos. Además, la temperatura óptima es de $28^{\circ} \mathrm{C}$ a $32^{\circ} \mathrm{C}$, si las condiciones internas de la colmena son favorables (DEFRA 2003).

La fase de pupa ocurre en el suelo; por tanto, el tipo y la humedad son factores que influyen en su desarrollo. Se menciona que prefiere suelos arenosos o de textura similar. Los que poseen exceso de humedad no son beneficiosos; los secos y duros tampoco son óptimos para su desarrollo, porque la pupa debe penetrar varios centímetros para completar el ciclo (Argueta 2015). 
Rev. Ciencias Veterinarias, Vol. 38, N² 2, [13-29], E-ISSN: 2215-4507, julio-diciembre, 2020

DOI: https://doi.org/10.15359/rcv.38-2.2

URL: https://www.revistas.una.ac.cr/index.php/veterinaria/index

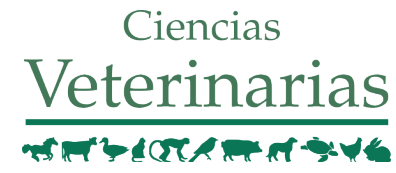

El escarabajo adulto evade la luz, por lo cual, el interior de la colmena es un espacio adecuado. Se ubica en los lugares más oscuros. Además, se protege del alcance de las obreras. Su actividad, para salir o entrar a la colonia, se intensifica en la noche (entre 8:00 a 10:00 pm) (Neumann \& Elzen 2004; Saldaña et al. 2014). De esta manera, evade a las abejas. Además, dificulta al apicultor su observación (Argueta 2015).

En cuanto a la sanidad de la colmena, se ha comprobado que colonias débiles (poca población de abejas) o con un nivel de estrés alto, por condiciones adversas (enfermedades como la Varroosis o la escasez de alimento), son más propensas a la invasión del escarabajo. Aunque, en una infestación masiva, cualquier colmena es susceptible, independiente de su manejo sanitario (Ellis 2003). Se debe mantener un manejo óptimo en los apiarios; realizar un control eficiente de plagas y enfermedades, así como proporcionar alimentación adecuada en la época lluviosa (Calderón et al. 2006; Saldaña et al. 2014). Abejas con alto comportamiento higiénico beneficiarán a la colmena, porque identifican celdas en las que el escarabajo ha depositado huevos y los eliminan. La detección, por parte de la abeja, ocurre al presentarse opérculos que han sido perforados por la hembra del escarabajo (Stedman 2006).

La alimentación de A. tumida es fundamental para su desarrollo. Se ha comprobado que, dependiendo de su fuente alimenticia, puede variar su comportamiento. Escarabajos que se han alimentado con proteína (huevos, larvas, pupas y polen) presentan una mayor descendencia, pero tienden a vivir menos. Mientras que aquellos con una dieta más concentrada en azúcares de fruta y miel, viven más tiempo, pero muestran una menor descendencia (Saldaña et al. 2014; Argueta 2015). El escarabajo adulto puede alimentarse y completar su ciclo en frutas en descomposición (melón, aguacate, mango y naranja) (Neumann \& Elzen 2004). Un aspecto por considerar, en su dispersión, es que los escarabajos adultos pueden volar y desplazarse a grandes distancias, junto a enjambres silvestres o en colonias utilizadas en la polinización de cultivos agrícolas; por lo cual, se debe implementar métodos de vigilancia en los apiarios para detectar su presencia.

\section{Materiales y métodos}

Con la finalidad de ampliar la información sobre A. tumida, se incluye una revisión de literatura sobre la distribución, biología y condiciones que benefician su desarrollo.

\section{Determinación de la presencia/ausencia del escarabajo}

Para evaluar la presencia y distribución del Pequeño Escarabajo en abejas africanizadas, en diferentes zonas apícolas de Costa Rica, se muestreó, durante el período de mayo a octubre del 2019, un total de 305 colmenas, distribuidas en 61 apiarios, ubicados en San Ramón, Alajuela y zonas aledañas (Figura 1). Este cantón del Valle Central de Costa Rica se caracteriza por su gran actividad apícola, la cual se considera como una de las principales actividades socioeconómicas de la región.

El muestreo de las colmenas se realizó de acuerdo con la metodología reportada en estudios anteriores (Ramírez \& Calderón 2018). En cada apiario, se analizó, aleatoriamente, cinco colmenas; en las cuales se revisó, minuciosamente, la tapa, los marcos, las cajas y el piso, con el fin de identificar la presencia de huevos, larvas y/o adultos del escarabajo. También, para reconocer algún signo relacionado, como galerías en los panales, apariencia húmeda o grasosa de las abejas y marcos, entre otros. 
Adicionalmente, se incluye el reporte de un escarabajo adulto, colectado de una colonia silvestre establecida dentro de una casa de habitación ubicada en Barreal, Ulloa de Heredia. Un espécimen fue remitido al laboratorio de Patología Apícola del Centro de Investigaciones Apícolas Tropicales (CINAT) de la Universidad Nacional (UNA), para su análisis e identificación.

Además, se muestreó un apiario, constituido por 30 colmenas, localizado en Potrerillos de Liberia, Guanacaste. Sobre todo, para tomar en cuenta la cercanía con el foco inicial de detección en la Cruz, Guanacaste (Figura 1). Al considerar que el apicultor informó sobre la posible presencia de escarabajos adultos. Se revisaron cinco colmenas, de las cuales se colectaron 10 especímenes adultos en un frasco con alcohol al 70.0\%, los cuales se analizaron en el laboratorio.

\section{Identificación del estadio adulto}

En el laboratorio, se analizaron escarabajos adultos, con el fin de reconocer sus características morfológicas específicas y realizar su identificación. Se revisó la forma de las antenas, el largo de los élitros y se midió su tamaño corporal.

\section{Resultados}

\section{Determinación de A. tumida en abejas africanizadas en el Valle Central.}

Se analizó un total de 305 colmenas, ubicadas en los distritos de: Piedades Sur, Piedades Norte, Zapotal, San Rafael, San Isidro, Concepción y San Juan, pertenecientes al cantón de San Ramón, Alajuela. Se realizó una revisión exhaustiva del interior de las colmenas, con el fin de detectar algún signo de $A$. tumida. Las 305 colmenas evaluadas resultaron negativas al Pequeño Escarabajo (Cuadro 1).

Cuadro 1. Revisión de colmenas $(n=305)$ para determinar la presencia/ausencia de A. tumida en San Ramón, Alajuela.

\begin{tabular}{cccc}
\hline Ubicación de los apiaries & Colmenas muestreadas & Lugar de revision & $\begin{array}{c}\text { Presencia de } \\
\boldsymbol{A} \text {. } \text { tumida } \boldsymbol{a}\end{array}$ \\
\hline Piedades Sur & 150 & Cámara de cría & Negativo \\
Piedades Norte & 5 & Cámara de cría & Negativo \\
Zapotal & 25 & Cámara de cría & Negativo \\
San Rafael & 40 & Cámara de cría & Negativo \\
San Isidro & 15 & Cámara de cría & Negativo \\
Concepción & 10 & Cámara de cría & Negativo \\
San Juan & 5 & Cámara de cría & Negativo \\
Otros & 55 & Cámara de cría & Negativo \\
\hline
\end{tabular}


Rev. Ciencias Veterinarias, Vol. 38, N² 2, [13-29], E-ISSN: 2215-4507, julio-diciembre, 2020

DOI: https://doi.org/10.15359/rcv.38-2.2

URL: https://www.revistas.una.ac.cr/index.php/veterinaria/index

Ciencias

Las colmenas evaluadas, en la zona apícola de San Ramón, resultaron negativas para el Pequeño Escarabajo. Sin embargo, es relevante indicar un hallazgo en el Valle Central del país. Se observó la presencia de escarabajos adultos en una colonia silvestre, establecida dentro de una casa de habitación en Barreal, Ulloa de Heredia, el 17 de setiembre del 2018. Un espécimen fue remitido al laboratorio de Patología Apícola (CINAT-UNA), para su análisis. Las características del escarabajo se detallan a continuación (Cuadro 2).

Cuadro 2. Análisis del escarabajo adulto proveniente de Barreal, Ulloa de Heredia.

\begin{tabular}{ccccc}
\hline Coloración & Tamaño $(\mathbf{m m})$ & Antenas & Alas (élitros) & Vellos \\
\hline Marrón oscuro (casi negro) & $\begin{array}{c}\text { Largo: } 6.0 \\
\text { Ancho: } 3.2\end{array}$ & Capitadas & $\begin{array}{c}\text { Más cortas que el } \\
\text { abdomen }\end{array}$ & Todo el cuerpo \\
\hline
\end{tabular}

Se debe resaltar las antenas ensanchadas en la parte distal, tipo capitadas y las alas más cortas que el abdomen, lo cual deja expuestos de 2 a 3 segmentos abdominales. Con base en las características morfológicas, las cuales concuerdan con las descritas en la literatura para el Pequeño Escarabajo (Stedman 2006; FERA 2013; Newman \& Ellis 2008) y la ubicación de colecta (en el interior de la colonia), se confirmó que el escarabajo analizado en el laboratorio corresponde a la especie A. tumida. Además, el Servicio Nacional de Salud Animal (SENASA) de Costa Rica comprobó, mediante análisis molecular de PCR, dicho hallazgo, confirmando la detección del escarabajo en el Valle Central de Costa Rica (Calderón \& Ramírez 2019).

Nueva detección del Pequeño Escarabajo en Guanacaste: En octubre de 2019, se determinó la presencia de escarabajos adultos en un apiario ubicado en Potrerillos de Liberia, Guanacaste. Se debe indicar que este apiario, constituido por 30 colmenas, se encuentra aproximadamente a $25 \mathrm{~km}$ del foco inicial de detección del Pequeño Escarabajo en Santa Cecilia, La Cruz de Guanacaste. Las características morfológicas, de los escarabajos adultos analizados, coincidieron con las descritas en la literatura para A. tumida, determinando una nueva detección en la provincia de Guanacaste (Cuadro 3 , Figura 1). Se debe mencionar que transcurrieron más de cuatro años para confirmar la presencia de este escarabajo en un nuevo sitio de la zona (agosto 2015 - octubre 2019), lo cual, probablemente, sugiere un desplazamiento natural.

\section{Distribución del Pequeño Escarabajo en abejas africanizadas en Costa Rica}

Se muestra la distribución de A. tumida, en el país, con base en los puntos geográficos indicados en este estudio, relacionados con la zona apícola muestreada y las detecciones confirmadas (Figura 1). Esta información permite observar su dispersión en colmenas de abejas africanizadas en Costa Rica.

\section{Características morfológicas de los escarabajos adultos}

Se indica las características morfológicas que diferencian el escarabajo A. tumida de otras especies de Nitidúlidos; por tanto, permiten su identificación. Con respecto a los escarabajos adultos (Figura 2), según Calderón et al. (2014), el Pequeño Escarabajo presenta una coloración marrón oscuro (casi negro), un tamaño promedio de $5.8 \mathrm{~mm}$ de largo y $3.3 \mathrm{~mm}$ de ancho. Las antenas son ensanchadas en la parte distal, lo cual se conoce como antenas capitadas (Ortiz, SF). Además, posee vellosidad en todo 
Cuadro 3. Características morfológicas de los escarabajos adultos $(n=10)$ procedentes de un apiario en Potrerillos de Liberia, Guanacaste.

\begin{tabular}{|c|c|c|c|c|c|}
\hline ID muestra & Coloración & Tamaño $(\mathrm{mm})$ & Antenas & Alas (élitros) & Vellos \\
\hline 1 & Marrón oscuro & $\begin{array}{l}\text { L: } 7.0 \\
\text { A: } 3.0\end{array}$ & Capitadas & ${ }^{\star}$ Cortas & Todo el cuerpo \\
\hline 2 & Marrón oscuro & $\begin{array}{l}\text { L: } 5.5 \\
\text { A: } 3.0\end{array}$ & Capitadas & ${ }^{\star}$ Cortas & Todo el cuerpo \\
\hline 3 & Marrón oscuro & $\begin{array}{l}\text { L: } 7.0 \\
\text { A: } 3.0\end{array}$ & Capitadas & ${ }^{\star}$ Cortas & Todo el cuerpo \\
\hline 4 & Marrón oscuro & $\begin{array}{l}\text { L: } 6.0 \\
\text { A: } 3.0\end{array}$ & Capitadas & ${ }^{\star}$ Cortas & Todo el cuerpo \\
\hline 5 & Marrón oscuro & $\begin{array}{l}\text { L: } 5.5 \\
\text { A: } 3.0\end{array}$ & Capitadas & ${ }^{\star}$ Cortas & Todo el cuerpo \\
\hline 6 & Marrón oscuro & $\begin{array}{l}\text { L: } 7.0 \\
\text { A: } 3.0\end{array}$ & Capitadas & ${ }^{*}$ Cortas & Todo el cuerpo \\
\hline 7 & Marrón oscuro & $\begin{array}{l}\text { L: } 5.5 \\
\text { A: } 3.0\end{array}$ & Capitadas & ${ }^{\star}$ Cortas & Todo el cuerpo \\
\hline 8 & Marrón oscuro & $\begin{array}{l}\text { L: } 5.5 \\
\text { A: } 3.0\end{array}$ & Capitadas & ${ }^{\star}$ Cortas & Todo el cuerpo \\
\hline 9 & Marrón oscuro & $\begin{array}{l}\text { L: } 6.0 \\
\text { A: } 3.0\end{array}$ & Capitadas & ${ }^{\star}$ Cortas & Todo el cuerpo \\
\hline 10 & Marrón oscuro & $\begin{array}{l}\text { L: } 6.0 \\
\text { A: } 3.0\end{array}$ & Capitadas & ${ }^{\star}$ Cortas & Todo el cuerpo \\
\hline
\end{tabular}

Abreviaturas: L: Largo, A: Ancho. ${ }^{\star}$ Cortas: Alas (élitros) más cortas que el abdomen.

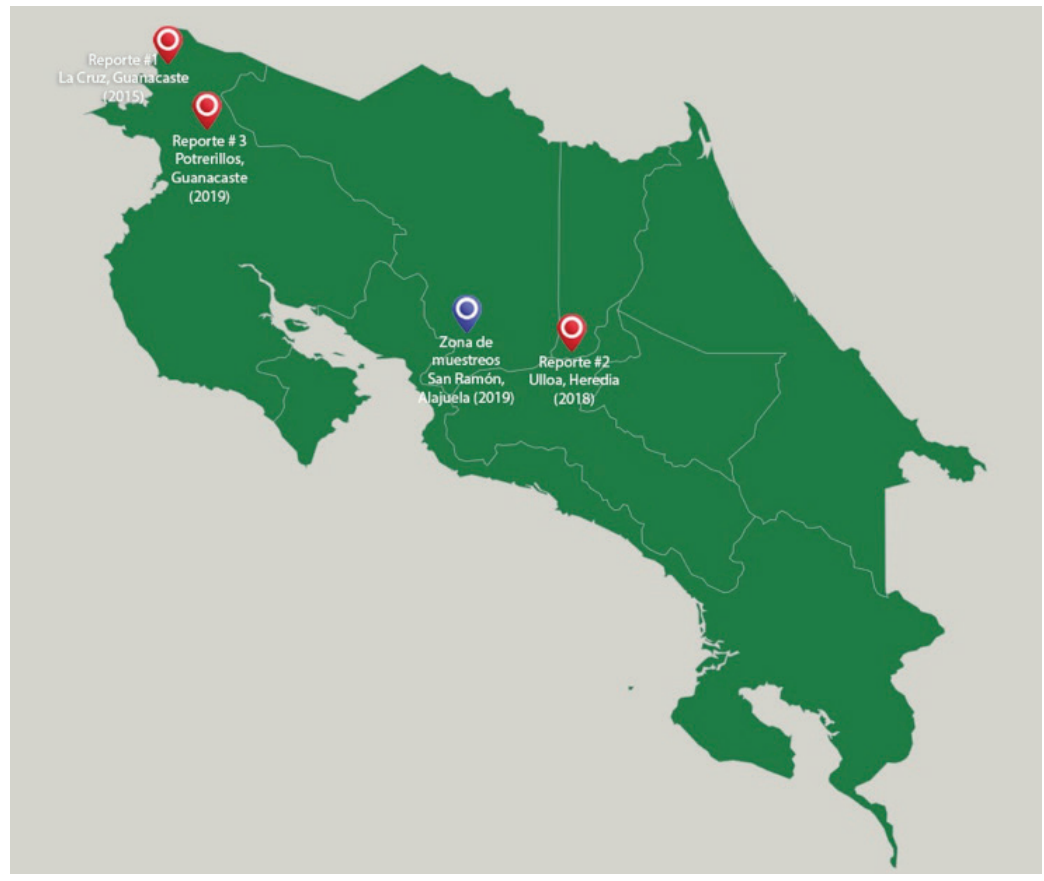

Figura 1. Zonas apícolas muestreadas y reportes sobre la presencia de A. tumida en colmenas de abejas africanizadas en Costa Rica. 


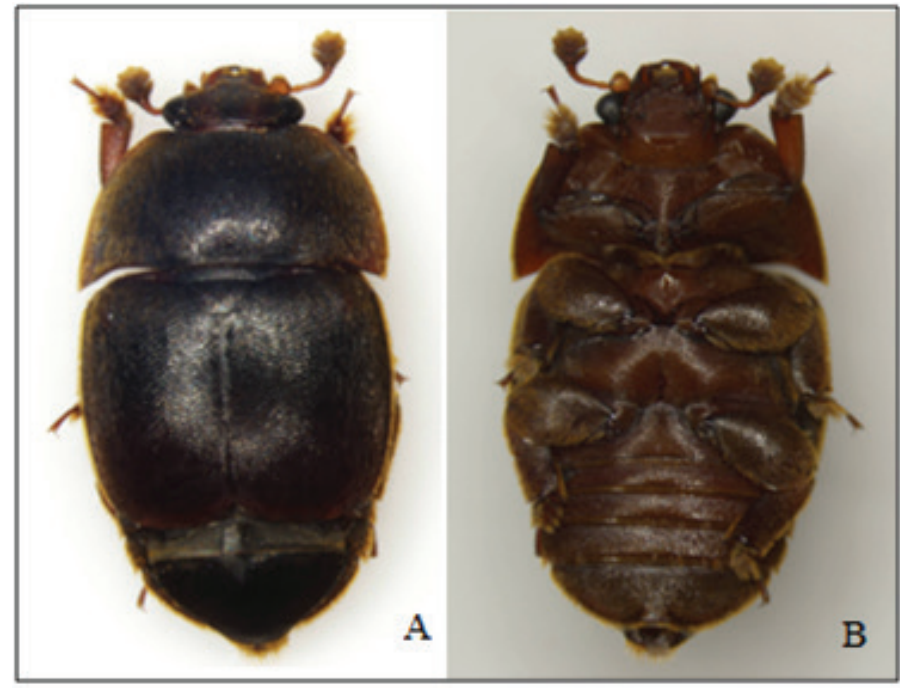

Figura 2. Vista dorsal (A) y ventral (B) de A. tumida (Ramírez \& Calderón 2018).

el cuerpo y algo muy característico de $A$. tumida son las alas, las cuales son más cortas que el abdomen, dejando expuestos, de 2 a 3 segmentos abdominales (Figura 2A) (Stedman 2006; Calderón et al. 2014). En una vista ventral del escarabajo, se puede resaltar que sus patas son robustas (Figura 2B).

\section{Discusión}

Como se indica, en marzo del 2014 se determinó la presencia de A. tumida en colmenas de abejas africanizadas en San Juan del Sur, Rivas de Nicaragua (Calderón et al. 2014), lo cual representó una amenaza de ingreso a Costa Rica, dado que el foco de detección se ubicó cerca de la región fronteriza (Figura 3A). A partir de esa fecha, se realizó un monitoreo del escarabajo en diferentes zonas apícolas del país. De esta manera, se confirmó, en agosto del 2015, la detección de A. tumida en Costa Rica. En un apiario centinela instalado por el SENASA en La Cruz, Guanacaste (Ramírez \& Calderón 2018). Dicho hallazgo se realizó más de un año después de su detección en Nicaragua, lo cual podría sugerir una dispersión natural del escarabajo, debido a la cercanía con el apiario infestado en Rivas (aproximadamente a $8.0 \mathrm{~km}$ ) (Figura 3B). Según Neumann et al. (2016), este escarabajo vuela activamente y es capaz de detectar colonias a una distancia entre 13 a $16 \mathrm{~km}$.

Monitoreo de colmenas en San Ramón: De acuerdo con los resultados obtenidos en investigación, en la cual las 305 colmenas, evaluadas en San Ramón, resultaron negativas a A. tumida. Se puede indicar que aún no se ha diseminado por la región del Valle Central. Dentro de los factores que, probablemente, han evitado esta dispersión, se encuentra el poco movimiento o traslado de colmenas a otras regiones del país. La mayoría de los productores indica que no realizan trashumancia de colmenas, al mantener los apiarios en su ubicación durante todo el año. Por otra parte, la producción de reinas se realiza a nivel local (algunos apicultores producen reinas para sus colmenas o las adquieren de criadores locales), lo cual disminuye el riesgo que representa introducirlas desde otras regiones. 


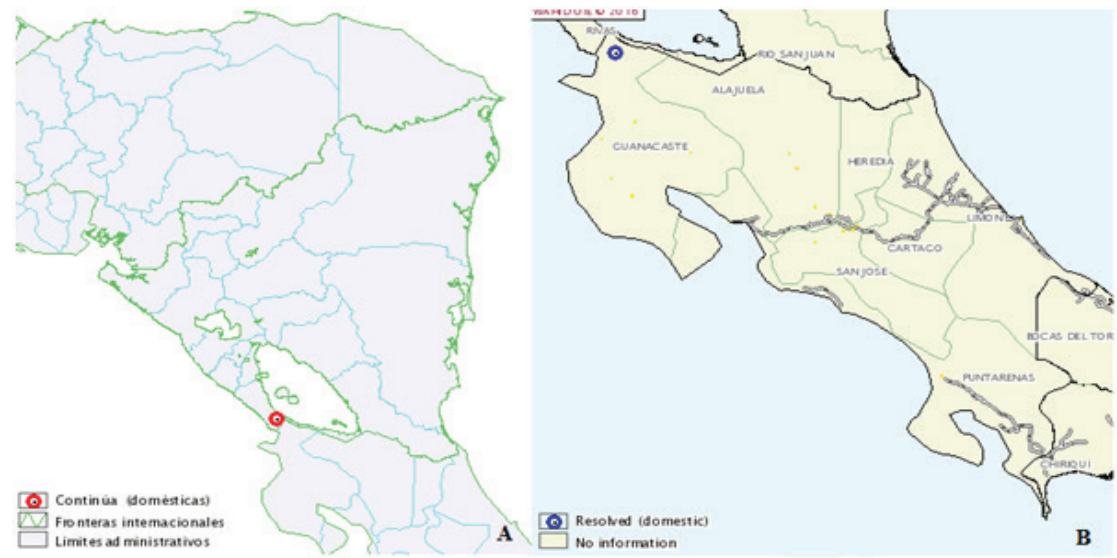

Figura 3. Confirmación del Pequeño Escarabajo en San Juan del Sur, Rivas Nicaragua (A), y en La Cruz, Guanacaste - Costa Rica (B) (OIE, 2014; OIE, 2015).

Además, se debe tomar en cuenta que la presencia de A. tumida en Costa Rica, no se ha manifestado de forma frecuente y severa. Probablemente, el comportamiento de la abeja africanizada, la cual predomina en los sistemas de producción apícola, ha evitado una amplia distribución en el país. Se ha reportado que, las abejas de tipo africanizado muestran cierta resistencia o tolerancia hacia este escarabajo (Elzen et al. 2001; Ellis et al. 2004; Spiewok \& Neumann 2006). Estudios realizados, en colmenas de abejas africanas, A. m. scutellata y A. m. capensis, no han indicado un impacto negativo de A. tumida (Ellis et al. 2003b; Neumann \& Härtel 2004; Amparán \& Amparán 2011).

Por otra parte, se ha realizado reportes de A. tumida afectando severamente colonias de abejas europeas (Elzen et al. 1999). Existen diferencias en el comportamiento defensivo e higiénico entre abejas de tipo europeo y africanizado (Ellis et al. 2003c). Se menciona la remoción de la colmena de larvas y huevos del escarabajo, así como un mayor hostigamiento que realizan las obreras hacia el adulto en abejas africanizadas (Neumann \& Härtel 2004; Spiewok \& Neumann 2006). Según han observado los apicultores sudafricanos, las abejas muestran un comportamiento defensivo contra el escarabajo adulto, mediante el aguijoneo (el cual muchas veces no resulta muy efectivo por la dureza del exoesqueleto del escarabajo), hasta su remoción y encapsulamiento. Además, por el comportamiento higiénico, la eliminación de larvas es más eficiente, en comparación con observaciones realizadas por apicultores de los Estados Unidos con abejas europeas, quienes no reportan este comportamiento en sus colmenas (Elzen et al. 2001).

Otro mecanismo de defensa, que utilizan las abejas melíferas, es el "encapsulamiento social” o también llamado "confinamiento", consistente en una estructura que construyen con base en propóleo y cera, para encerrar los escarabajos; con ello limitar su reproducción dentro de la colmena (Elzen et al. 2001). Este comportamiento se realiza tanto en abejas de origen europeo como africanizado (Ellis et al. 2004). No obstante, se menciona que este comportamiento es más desarrollado y eficiente en abejas de tipo africanizado, dado que, en las europeas resulta eficiente solo cuando la población de escarabajos es baja, mientras que, en infestaciones severas, el encapsulamiento no funciona como mecanismo de control (Ellis et al. 2003a).

Presencia de A. tumida en abejas melíferas del Valle Central: De acuerdo con la distribución del 
Rev. Ciencias Veterinarias, Vol. 38, N 2, [13-29], E-ISSN: 2215-4507, julio-diciembre, 2020

DOI: https://doi.org/10.15359/rcv.38-2.2

URL: https://www.revistas.una.ac.cr/index.php/veterinaria/index

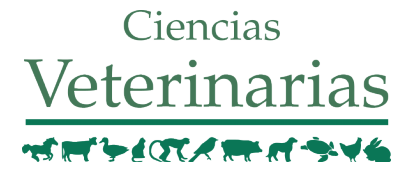

escarabajo en Costa Rica, se debe indicar que, tres años después de su primera detección, se determinó su presencia en una colonia silvestre en una zona urbana de Ulloa, Heredia (Calderón \& Ramírez 2019). Se desconoce, con exactitud, la procedencia de estos escarabajos. Se debe mencionar que no aparecen registradas colmenas y/o apiarios en los alrededores del sector. Sin embargo, aproximadamente a $1.0 \mathrm{~km}$ del sitio, se encuentra el Centro Nacional de Abastecimiento y Distribución de Alimentos (CENADA), el cual es un mercado mayorista dedicado al comercio y distribución de productos hortofrutícolas, alimentos frescos y flores, a diferentes mercados del país (PIMA/CENADA 2020). La mayoría de los productos que se trasiegan en el CENADA y que proceden de distintas partes del país, entre ellas Guanacaste, representan posibilidades de dispersión y desarrollo para el Pequeño Escarabajo, pues se conoce que puede utilizar frutas como fuente alternativa de alimento y reproducción, especialmente en ausencia de colonias de abejas melíferas (Neumann \& Elzen 2004). A. tumida se ha reportado en frutas como: aguacate, melón, piña y banano (Neumann \& Elzen 2004; Cuthbertson et al. 2013; FERA 2015). En general, se menciona que, dentro de las fuentes de alimento de los escarabajos de la familia Nitidulidae, se encuentran las frutas frescas o en descomposición (Solís 2002).

Por otra parte, se debe tomar en cuenta la capacidad de A. tumida de establecerse en colonias de otras especies de abejas. Entre ellas, las abejas nativas sin aguijón, como: Melipona beecheii, Dactylurina staudingerii, Trigona carbonaria (Lóriga et al. 2014) y Austroplebeia australis (Halcroft et al. 2011). Al considerar que la meliponicultura es una actividad realizada en diferentes regiones costarricenses, se debe prestar atención al posible desarrollo del escarabajo en estas abejas.

Otra posibilidad de diseminación lo constituye el comercio de abejas y sus productos. En el informe científico, sobre el comercio de cera de abeja, se relacionó el ADN mitocondrial (COIgen, n= 296 individuos de $A$. tumida en 98 ubicaciones y reveló 90 haplotipos) con respecto a los datos de comercio de cera (FAO) publicados para 12 invasiones de A. tumida. En nueve de los 12 casos de invasión, los datos genéticos y de comercio de cera de abeja coinciden (Idrissou et al. 2019). Estos resultados indican que las importaciones de: Etiopía, Sudáfrica, Tanzania y Estados Unidos han sido las principales responsables de la diseminación del escarabajo a diferentes partes del mundo. En los análisis de ADN mitocondrial, se encontró, para Costa Rica, el haplotipo 23 (Foco: Guanacaste, 2016), el mismo para Canadá, Estados Unidos y Corea del Sur. Durante el período del 2012 al 2014, Costa Rica importó cera de Estados Unidos (1.33 toneladas) y de Argentina (2 toneladas) (Idrissou et al. 2019). Lo cual sugiere que la importación y comercio de cera, procedente de Estados Unidos, es una fuente probable de propagación del escarabajo en Costa Rica (Idrissou et al. 2019). Por consiguiente, se debe resaltar la importancia de la trazabilidad de los productos de la colmena, para identificar el origen de una plaga y su distribución, pues la importación ilegal puede tener resultados negativos para el sector apícola.

Nueva detección en Guanacaste: Un nuevo hallazgo de A. tumida se realizó en un apiario ubicado en Liberia de Guanacaste, a una distancia aproximada de $25 \mathrm{~km}$ del foco inicial. Dicha detección se efectuó más de cuatro años después de su confirmación en la Cruz (agosto 2015 - octubre 2019). En este caso, al analizar la distancia respecto al foco inicial, el tiempo transcurrido, desde su detección y que A. tumida es un volador activo, que acompaña enjambres silvestres, podría considerarse la posibilidad de una dispersión natural. De acuerdo con Neumann et al. (2016), este escarabajo es capaz de detectar colonias a una distancia entre 13 a $16 \mathrm{~km}$. Sin embargo, se desconoce, con certeza, cuáles mecanismos intervienen para que expanda su territorio (Spiewok et al. 2007). 
Mediante el estudio de la biología del escarabajo, se podría identificar el "modus operandi" tan exitoso de adaptabilidad y capacidad de dispersión. Según Kollman et al. (2016), la supervivencia y el éxito reproductivo de un parásito que vive en una colmena, en este caso A. tumida, depende, en gran medida, de la capacidad de comprender, al menos una parte de la comunicación química de su hospedero. Está reportado que el Pequeño Escarabajo posee un lóbulo antenal (que recibe información de quimiorreceptores de las antenas y aparato bucal) de mayor tamaño que otros coleópteros (Kollman et al. 2016). El sentido del olfato es fundamental para la subsistencia y búsqueda de hospederos por parte de los escarabajos adultos, los cuales son atraídos por fuentes alimenticias fermentadas (Neumann \& Elzen 2004). La fermentación de la miel, por la acción de la levadura K. omheri, presente en las excretas de las larvas de A. tumida, genera ácidos volátiles que imitan las feromonas de las abejas y atraen más escarabajos a la colonia (Tauber et al. 2019). Lo anterior, representa uno de los principales mecanismos de adaptación del escarabajo a las colmenas de abejas melíferas.

Por otra parte, se ha propuesto que el cambio climático ha influido en el desarrollo de A. tumida, dado que el estadio de pupa se completa en el suelo y su éxito depende de la temperatura del suelo y la humedad, los cuales han sido alterados por el cambio climático (calentamiento global). En un estudio se evidencia que, al aumentar la temperatura en el suelo, el desarrollo de la fase de pupa a adulto se acelera, lo cual incrementa la tasa de supervivencia. Esto sugiere un alto riesgo de invasión de apiarios cercanos al foco inicial y la infestación de colonias de abejas silvestres (Cornelissen et al. 2019).

Diagnóstico diferencial con escarabajos adultos de otras especies: En Costa Rica, están descritas, en la familia Nitidulidae, algunas especies de escarabajos saprófitos, con una apariencia similar a A. tumida, por lo cual, pueden confundirse al encontrarse en las colmenas de abejas. Entre estos escarabajos se puede mencionar las especies: Lobiopa insularis (Coleoptera: Nitidulidae: Nitidulinae) (Figura 4A) y Aethina villosa (Coleoptera: Nitidulidae) (Figura 4B). Una característica que permite orientar el diagnóstico diferencial es que los escarabajos L. insularis y A. villosa, poseen alas largas, las cuales cubren completamente el abdomen (Figura 4), mientras que en A. tumida, las alas son cortas, dejando expuestos de 2 a 3 segmentos abdominales (Figura 2).

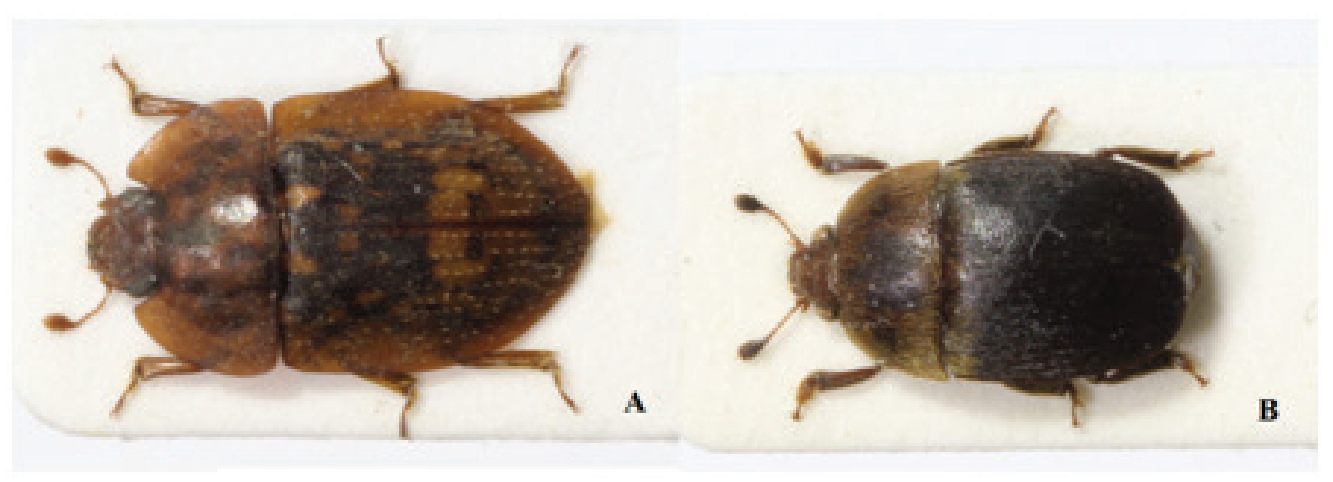

Figura 4. Escarabajos que podrían confundirse con el Pequeño Escarabajo de la Colmena. Lobiopa insularis (A) y Aethina villosa (B) (Solís 2017). 
En julio del 2014, se colectaron escarabajos adultos de una colmena que evadió (abandonó el nido de cría) en un apiario ubicado en Vuelta de Jorco, Aserrí de San José. No se observó larvas, ni otros estadios inmaduros. Estos escarabajos se confirmaron como A. villosa (colección de referencia del INBio, A. Solís). La especie $A$. villosa está poco descrita en la literatura. Se menciona que se encuentra distribuida en México y Centroamérica (A. Solís, comunicación personal.). Además, se identificó algunos escarabajos del género Lobiopa sp. No obstante, estos escarabajos no se consideran una amenaza para las abejas, debido a que no poseen adaptaciones morfológicas ni de comportamiento para integrarse al entorno de la colonia. Probablemente, invaden la colmena de manera accidental, en busca de alimento en los detritos, pues se nutren de sustratos fúngicos, savia de las plantas; también, pueden encontrarse en las flores (Blake 2011). Sin embargo, existen reportes de otros escarabajos nitidúlidos, tanto en $A$. mellifera, como en colonias de abejas sin aguijón, en las cuales se han observado daños (Krishnan et al. 2015). Específicamente, se señala que Haptoncus luteolus (plaga de frutas en Malasia), parasita colonias de abejas nativas como: Tetragonula laeviceps, Trigona thoracica y Heterotrigona itama; lo cual, sugiere que puede afectar diferentes especies de abejas sociales, similar al Pequeño Escarabajo de la Colmena (Krishnan et al. 2015). Por el contrario, la mayoría de los escarabajos solo buscan refugio y/o comida (restos de polen) en las colonias de abejas, como ocurre comúnmente con C. luteus en Europa (Neumann \& Ritter 2004) y L. insularis y A. villosa en nuestra región.

En conclusión, con base en los datos analizados en este estudio, se puede indicar la ausencia de $A$. tumida en colmenas de abejas africanizadas en la región apícola de San Ramón. Por otra parte, se determinó la presencia de este escarabajo en una colonia silvestre, ubicada en una zona urbana del distrito de Ulloa, Heredia. Además, se realizó un nuevo hallazgo de escarabajos adultos en la provincia de Guanacaste, específicamente en un apiario ubicado en Potrerillos de Liberia.

Perspectivas: Los resultados sobre la distribución del Pequeño Escarabajo en colmenas de abejas africanizadas, realizados en La Cruz, Guanacaste en el 2015; en Ulloa de Heredia en el 2018 y recientemente en la zona de Potrerillos de Liberia, Guanacaste, en 2019. En todos se determinó la presencia de escarabajos adultos; no obstante, no han evidenciado un impacto negativo sobre la condición sanitaria de las colmenas. Algunos autores sugieren que las poblaciones de A. tumida, de recién ingreso, necesitan adaptarse al ambiente, para reproducirse y aumentar en cantidad, antes de ocasionar daños a las colmenas (Idrissou et al. 2019). Por tanto, es recomendable continuar la investigación de su dispersión en el país e implementar estudios sobre la dinámica poblacional y el impacto en la condición general de las colmenas. Lo cual, permitirá describir y cuantificar los efectos que pueda causar $A$. tumida en colmenas de abejas africanizadas en condiciones tropicales.

\section{Agradecimientos}

Agradecer a los apicultores de las diferentes zonas apícolas de Costa Rica, especialmente los de San Ramón, por permitir la inspección y el muestreo de colmenas para la detección del Pequeño Escarabajo de la Colmena. Además, agradecemos a nuestro compañero M.Sc. Fernando Ramírez Arias (CINATUNA) por colaborar activamente en la colecta de escarabajos adultos. Un agradecimiento especial al Ing. Juan Vicente Orozco Delgado (MAG-San Ramón) por el trabajo y apoyo brindado durante la revisión de colmenas. 


\section{Referencias}

Amparán, D. \& Amparán, L. 2011. El pequeño escarabajo de la colmena Aethina tumida Murray (Coleoptera: Nitidulidae) biología y control. Epidemiología de enfermedades parasitarias en animales domésticos 30: 455-467.

Argueta, A. 2015. Determinación de la presencia de tres ectoparásitos en la abeja (Apis mellifera) en el área de acción de la cooperativa de apicultores del suroccidente de Guatemala, en Quetzaltenango. Licenciatura en Zootecnia, Guatemala.

Blake, E. 2011. Investigating the integration of small hive beetles (Aethina tumida Murray, Coleoptera: Nitidulidae) into western honey bee (Apis mellifera L., Hymenoptera: Apidae) colonies. Requirements for the degree of Doctor of Philosophy. University of Florida, USA.

Calderón, R.A., Arce, H. \& Ramírez, J.F. 2006. El Pequeño Escarabajo de la Colmena Aethina tumida Murray, un problema importante que afecta a las abejas melíferas. Rev. Ciencias Veterinarias 24(1): 49-55. https://www.revistas.una.ac.cr/index.php/ veterinaria/article/view/4757.

Calderón, R.A., Ramírez, M., Ramírez, J.F. \& Villagra, W. 2014. Primer reporte de la presencia del Pequeño Escarabajo de la Colmena Aethina tumida, en colmenas de abejas africanizadas en Nicaragua. Rev. Ciencias Veterinaria 32(1): 29-33. http://www.revistas.una.ac.cr/index.php/veterinaria/index.

Calderón, R.A. \& Ramírez, M. 2019. New Record of the Small Hive Beetle, Aethina tumida, in Africanized Honey Bee Colonies in Costa Rica. Bee World 96(3): 87-89. doi.10.1080/0005772X.2019.1579294.

Calvo, M., Balart, M., Baz, A., Creus, S., Dueso, M. \& Farré, C. 2013. Principales enfermedades de etiología vírica de interés en apicultura.

Cornelissen, B., Neumann, P. \& Schweiger, O. 2019. Global warming promotes biological invasion of a honey bee pest. Glob. Chang. Biol. 25: 3642-3655. https://doi.org/10.1111/gcb.1479.

Cuthbertson, A., Wakefield, M., Powell, M., Marris, G., Anderson, H., Budge, G., Mathers, J., Blackburn, L. \& Brown, M. 2013. The small hive beetle Aethina tumida: A review of its biology and control measures. Curr. Zool. 59(5): 644-653. https://doi-org.una.idm.oclc.org/10.1093/czoolo/59.5.644.

DEFRA. 2003. The Small Hive Beetle: a serious new threat to European apiculture. International Bee Research Association (IBRA), Cardiff, UK.

Ellis, J. 2003. Ecology and Control of Small Hive Beetles (Aethina tumida Murray). (Tesis de Doctorado). Rhodes University, Grahamstown, Sudáfrica.

Ellis, J., Hepburn, H., Ellis, A. \& Elzen, P. 2003a. Social encapsulation of the small hive beetle (Aethina tumida Murray) by European honeybees (Apis mellifera L.) Insectes Soc. 50: 286-291. https://doi. org/10.1007/s00040-003-0671-7.

Ellis, J., Holland, A., Hepburn, R., Neumann, P. \& Elzen, P. 2003b. Cape (Apis mellifera capensis) and European (Apis mellifera) honey bee guard age and duration of guarding small hive beetles (Aethina tumida). J. Apic. Res. 42(3): 32-34. https://doi.org/10.1080/00218839.2003.11101085.

Ellis, J., Hepburn, R., Delaplane, K., Neumann, P. \& Elzen, P. 2003c. The effects of adult small hive beetles, Aethina tumida (Coleoptera: Nitidulidae), on nests and flight activity of Cape and European honey bees (Apis mellifera). Apidologie 34: 399-408. doi.10.1051/apido:2003038. 
Rev. Ciencias Veterinarias, Vol. 38, N² 2, [13-29], E-ISSN: 2215-4507, julio-diciembre, 2020

DOI: https://doi.org/10.15359/rcv.38-2.2

URL: https://www.revistas.una.ac.cr/index.php/veterinaria/index

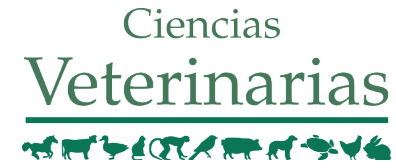

Ellis, J., Hepburn, R. \& Elzen, P. 2004. Confinement of small hive beetles (Aethina tumida) by Cape honeybees (Apis mellifera capensis). Apidologie 35: 389-396. doi: 10.1051/apido:2004030.

Ellis, M., Ellis, J. \& Hodges, C. 2007. Small Hive Beetle Aethina tumida Murray: Nitidulidae: Coleoptera. Florida Department of Agriculture and Consumer Services, Department of Entomology, North Carolina State. Publication Number 0018.

Elzen, P., Baxter, J., Westervelt, D., Randall, Ch., Delaplane, K., Cutts, L. \& Wilson, W. 1999. Field control and biology studies of a new pest species, Aethina tumida Murray (Coleoptera, Nitidulidae), attacking European honey bees in the Western Hemisphere. Apidologie 30: 361-366. doi.10.1051/apido:19990501.

Elzen, P., Baxter, J., Neumann, P., Solbring, A., Pirk, C., Hepburn, H. \& Westervelt, D. 2001. Behaviour of african and european subspecies of Apis mellifera toward the small hive beetle, Aethina tumida. J. Apic. Res. 40: 40-41. https://doi.org/10.1080/00218839.2001.11101049.

Faucon, J.P. 2003. El pequeño coleóptero Aethina tumida nueva e inquietante progresión?. Santé de I’Abeille. F-04500 Riez-France, Unité Pathologie de I’Abeille (France). http://www.beekeeping. org/sante- delabeille/) articles/pequeno coleoptero.htm.

Food and Environment Research Agency (FERA). 2015. The Small Hive Beetle a serious threat to European apiculture. http://www.nationalbeeunit.com/download Document.cfm?id=17.

Halcroft, M., Spooner-Hart, R. \& Neumann, P. 2011. Behavioral defense strategies of the stingless bee, Austroplebeia australis, against the small hive beetle, Aethina tumida. Springer 58: 245-253. https://doi.org/10.1007/s00040-010-0142.

Hayes, R., Rice, S., Amos, B. \& Leemon, D. 2015. Increased attractiveness of honeybee hive product volatiles to adult small hive beetle, Aethina tumida, resulting from small hive beetle larval infestation. Entomol. Exp. Appl. 155(3): 240-248. https://doi-org.una.idm.oclc.org/10.1111/eea.12304.

Hepburn, H. \& Radloff, S. 1998. Honeybees of Africa. Springer-Verlag Berlin Heidelberg New York.

Idrissou, F., Huang, Q., Yañez, O. \& Neumann, P. 2019. International beeswax trade facilitates small hive beetle invasions. Scientific Reports 9(1): 1-10. https://doi-org.una.idm.oclc.org/ 10.1038/ s41598-019-47107-6.

Krishnan, K., Neumann, P., Ahmad, A. \& Pimid, M. 2015. A scientific note on the association of Haptoncus luteolus (Coleoptera: Nitidulidae) with colonies of multiple stingless bee species. Apidologie 46: 262-264. doi.10.1007/s13592-014-0312-3.

Kollmann, M., Rupenthal, A., Neumann, P., Huetteroth, W. \& Schachtner, J. 2016. Novel antennal lobe substructures revealed in the small hive beetle Aethina tumida. Cell Tissue Res. 363(3): 679-692. https://doi.org/10.1007/s00441-015-2282-9.

Lóriga, W., Fonte, L. \& Demedio, J. 2014. Reporte de Aethina tumida Murray (Coleoptera, Nitidulidae) en colonias de la abeja sin aguijón Melipona beecheii Bennett de Matanzas y Mayabeque. Rev. Salud Anim. 36(3): 201-204.

Martínez, M., Rosas, J., Prieto, D., Carmona, A., Peña, B. \& Ávila, F. 2016. Presencia de Varroa destructor, Nosema apis y Acarapis woodi en abejas (Apis mellifera) de la región oriente del estado de México. Mayo-Agosto 6(2): 30-38. http://dx.doi.org/10.21929/abavet 2016.62.3. 
Ministerio de Agricultura y Ganadería (MAG-CR). 2012. Protocolo de atención a brotes de Aethina tumida. Heredia. http://www.senasa.go.cr/senasa/sitio/files/18071207 4329.

Neumann, P. 2004. The Small Hive Beetle (Aethina tumida Murray, Coleoptera: Nitidulidae). Tesis doctoral: Parasitismo inter e intraespecífico en abejas (Apis mellifera L.): el Pequeño Escarabajo de la Colmena (Aethina tumida Murray) y la abeja del Cabo (A. m. capensis Esch). https://sundoc. bibliothek.uni-halle.de/habil-online/04/05H007/ habil.pdf.

Neumann, P. \& Elzen, J. 2004. The biology of the small hive beetle (Aethina tumida, Coleoptera: Nitidulidae): Gaps in our knowledge of an invasive species. Apidologie 35(3): 229-247. https://doi. org/10.1051/apido:2004010.

Neumann, P. \& Hartel, S. 2004. Removal of small hive beetle (Aethina tumida) eggs and larvae by African honeybee colonies (Apis mellifera scutellata). Apidologie 35: 31-36. doi.10.1051/apido:2003058.

Neumann, P. \& Ritter, W. 2004. A scientific note on the association of Cychramus luteus (Coleoptera: Nitidulidae) with honeybee (Apis mellifera) colonies. Apidologie 35: 665-666. https://doi. org/10.1051/apido:2004051.

Neumann, P. \& Ellis, J. 2008. The small hive beetle (Aethina tumida Murray, Coleoptera: Nitidulidae): distribution, biology and control of an invasive species. J. Apic. Res. and Bee World 47(3): 181-183. https://doi.org/10.1080/00218839.2008.11101453.

Neumann, P., Pettis, J. \& Schäfer, M. 2016. Quo vadis Aethina tumida? Biology and control of small hive beetles. Apidologie 47(3): 427-466. https://doi-org.una.idm.oclc.org/10.1007 /s13592-016-0426-x.

Neumann, P., Spiewok, S., Pettis, J., Radloff, S.E., Spooner-Hart, R. \& Hepburn, R. 2018. Differences in absconding between African and European honeybee subspecies facilitate invasion success of small hive beetles. Apidologie 49(5): 527-537. https://doi-org.una.idm.oclc.org/10.1007/s13592018-0580-4.

Organización Mundial de Sanidad Animal (OIE). 2013. Infestación por el escarabajo de las colmenas (Aethina tumida), El Salvador. https://www.oie.int/wahis 2/public/ wahid.php/Reviewreport/Re view? pagerefer=MapFullEventReport\&reportid=14498.

Organización Mundial de Sanidad Animal (OIE). 2014. Infestación por el escarabajo de las colmenas (Aethina tumida), Nicaragua. https://www.oie.int/wahis 2/public/ wahid.php/Reviewreport/Re view?pagerefer $=$ MapFullEventReport\&reportid $=14888$.

Organización Mundial de Sanidad Animal (OIE). 2015. Infestación por el escarabajo de las colmenas (Aethina tumida), Costa Rica. https://www.oie.int/wahis 2/public/ wahid.php/Reviewreport/ Review?page refer=MapFullEventReport\&reportid=18506.

Organización Mundial de Sanidad Animal (OIE). 2016. Infestación por el escarabajo de las colmenas (Aethina tumida), Brasil. https://www.oie.int/wahis 2/public/wahid.php/Reviewreport/ $\underline{\text { Review?page refer=MapFullEventReport\&reportid=19529\&newlang=es. }}$

Ortiz, W. (SF). Entomología general. Instituto de educación superior tecnológico público. http:// isthuando.edu.pe/archivos/entomologia.pdf. 
Programa Integral de Mercadeo Agropecuario (PIMA). Centro Nacional de Abastecimiento y Distribución de Alimentos (CENADA). 2020. CENADA: objetivos y servicios. http://www.pima. go.cr/cenada/.

Ramírez, M. \& Calderón, R.A. 2018. Situación del Pequeño Escarabajo, Aethina tumida, en colmenas de abejas africanizadas (Apis mellifera) en Costa Rica: Muestreo de apiarios 2014-2017. Rev. Ciencias Veterinarias 36(1): 19-96. doi.10.15359/rcv.36-1.2.

Roldán, J. 2014. Detección de Aethina tumida, Nosemiasis y Varroa destructor en las abejas melíferas de la Comarca Lagunera. Torreón, Coahuila, México.

Saldaña, L., Lara, L. \& Dorantes, L. 2014. Nuevos Manejos en la Apicultura para el Control del Pequeño Escarabajo de la Colmena Aethina tumida Murray. México.

Sanford, M. 2005. Featured Creatures. Small hive beetle: Aethina tumida (Murray) (Insecta: Coleoptera: Nitidulidae). University of Florida, USA. http://www.entnemdept.ufl. edu/creatures/index.htm.

Solís, A. 2002. Escarabajos de Costa Rica: las familias y subfamilias más comunes. Instituto Nacional de Biodiversidad (INBio): Santo Domingo, Heredia, Costa Rica.

Solís, A. 2017. Las familias de Coleoptera del área de Conservación Guanacaste. https://www. acguanacaste.ac.cr/investigacion/coleoptera-de-acg\#Nitid.

Spiewok, S. \& Neumann, P. 2006. The impact of recent queen loss and colony phenotype on the removal of small hive beetle (Aethina tumida Murray) eggs and larvae by african honeybee colonies (Apis mellifera capensis). J. Insect Behav. 5(19): 601-611. https://doi.org/10.1007/s10905-006-9046-Z.

Spiewok, S., Duncan, M., Spooner-Hart, R., Pettis, J. \& Neumann, P. 2007. Small hive beetle, Aethina tumida, populations II: Dispersal of small hive beetles. Apidologie 39: 683-693. https://doi. org/10.1051/apido:2008054.

Stedman, M. 2006. Small Hive Beetle (SHB): Aethina tumida Murray (Coleoptera: Nitidulidae). Goverment of South Australia: Primary Industries and Resources. http://www.cvbclub.com/ images/SHB-Australia\%202006.pdf.

Tauber, J., Childers, A. \& Evans, J. 2019. Draft genome sequence of the yeast Kodamaea ohmeri, a symbiont of the small hive beetle. Microbiol. Resour. Announc. 8: 1-3. https:/doi.org/10.1128/ MRA.00450-19.

Torto, B., Arbogast, R., Engelsdorp, D., Willms, S., Purcell, D., Boucias, D., Tumlison, J. \& Teal, P. 2007. Trapping of Aethina tumida Murray (Coleoptera: Nitidulidae) from Apis mellifera L. (Hymenoptera: Apidae) Colonies with an In-Hive Baited Trap. J. Chem. Ecol. 5: 1018-1024. doi.1 0.1603/0046225X(2007)36[1018:TOATMC]2.0. CO;2.

Villalobos, E., Nikaido, S., Wong, J., Higes, M., Calderón, R.A. \& Ramírez, M. 2014. Biología y recomendaciones de manejo para el Pequeño Escarabajo de la Colmena (Aethina tumida). UH Honeybee Project. 